\title{
Theoretical analysis of operator actions when driving vehicle
}

\author{
V.S. Shkrabak ${ }^{1, *}$, E.N. Khristoforov ${ }^{2}$, N.E. Sakovich ${ }^{2}$, A.A. Popov ${ }^{1}$, R.V. Shkrabak ${ }^{1}$, and \\ P.P. Grigorov ${ }^{1}$ \\ ${ }^{1}$ St. Petersburg State Agrarian University, Department of Safety of Technological Processes and \\ Production, St. Petersburg, Russia \\ ${ }^{2}$ Bryansk State Agrarian University, Department of Safety and Environmental Engineering, Bryansk \\ Russia
}

\begin{abstract}
Approaches to the process of vehicle research are described, research methods are presented, their advantages and disadvantages, and the important role of mathematical research is noted. The points of differences between operator control and automatic control systems are considered. A mathematical model of the operator's actions in driving a vehicle is described, which reflects the psychophysical properties of the operator, the presence of absolute and differential thresholds of sensitivity. The model takes into account the delay in the operator's response and the discreteness of information perception. The model can be used for modeling all software management tasks. Moreover, when forming the control action of the operator - in the mathematical model, the characteristic features of the operator's activity that differ from an automatic control system are considered, and when forming the control action of the operator - in the mathematical model, some conditions were applied, thanks to which, it is possible to calculate the necessary deviation of the controls, such as the steering wheel and, accordingly, the wheels of the vehicle, knowing the required nature of changes in the parameters of the traffic's vehicle.
\end{abstract}

\section{Introduction}

The problems of the prevention of road accidents (RA) are well known to be global, as they annually kill hundreds of thousands of people and injure millions. The global nature of the problem began with the widespread motorization of society and lasts to the present. It is enough to note that in our country 50-55 people in average die almost every day in road traffic accidents, and about 700 people are injured.

In the last decade, a number of organizational and regulatory measures have been taken at the government level, which have allowed substantially (almost $1.5-1.7$ times) to reduce the number of people killed and injured in road accidents, as well as the number of road accidents themselves. Despite this, many issues of the problem of preventing accidents are not fully resolved to date. This is due to the fact that the problem concerns a complex

\footnotetext{
* Corresponding author: v.shkrabak@mail.ru
} 
biotechnical system of interaction between people and mobile equipment. There are also psycho-physiological aspects of the work related to the human factor.

Such a situation in the interests of further reducing the number of accidents and their consequences makes one continue to search for ways solving the problem intensively. At present, five priority ways are characteristic to solve the problem, namely: improving the regulatory framework; increasing the level of engineering and technical safety of vehicles; personnel aspects of the problem (in a broad sense, including the human factor); quality of the road network and traffic management; scientific support of the problem.

\section{Object of research}

This article is devoted to one of the most important preventive measures, that is the theoretical analysis of the operator actions when driving the vehicle. This is due to the fact that a decisive element in difficult traffic situations affecting the outcome of events preceding incidents or accidents is the operator - driver with his abilities to assess the multifactor situation almost instantly and make management decisions that exclude incidents. At the same time, the situation is aggravated by the fact that the behaviour of traffic participants is characterized by uncertainty described by the provisions of the probability theory. In this case, the operator - the driver, being in a limited space - the cabin does not have the possibility of a circular view and time for it.

\section{Research methods}

According to the degree of reliability of studies of the closed loop control properties "the operator-the vehicle" the following methods of researches can be distinguished: road tests, researches on modeling operational complexes; researches using mathematical modeling on a computer [3-6].

The most reliable results are obtained in road tests. However, such researches have significant drawbacks. The main ones among them are as such:

- the very high cost of experiments associated with the need to create experimental vehicles, the expenditure of their resource, fuel, etc.;

- the danger of experimentation in road tests;

- limited possibility of varying the vehicle's performance, its stability and controllability characteristics.

The researches of the properties of the "operator-vehicle" loop on the computer are free from these shortcomings, but they give less accurate results, since it is not the phenomenon itself that is investigated, but its mathematical model. Therefore, the modelling results must necessarily be verified experimentally, since the experiment performs the function of a criterion for the scientific knowledge truth in general. In this case, road tests can be carried out only to check adequacy of the mathematical model of the simulated process, and the researches should be carried out mainly on a computer. This combination of research methods contains great opportunities.

The more precise the mathematical model describes the properties of the "operatorvehicle" loop, the more reliable the results are obtained on a computer. If the development of the mathematical model of a vehicle does not cause fundamental difficulties, then the mathematical description of the operator actions is associated with serious difficulties. The complexity of describing the psychophysical characteristics of a person (randomness, discreteness, nonlinearity, non-stationarity of his actions) does not allow to obtain a universal mathematical model of the operator actions. However, when considering specific 
problems, it is possible to obtain partial mathematical models that describe the actions of the operator in different situations quite correctly [6-7].

The first attempts of mathematical description of the operator actions came down to replacing the operator by some autopilot with amplification constant. At the same time, as I.E. Tsibulevsny figuratively showed, the specific features of the control system of movements in a living organism were overlooked and the fact that this system is as complex as the most complex technical control system, as a modern computer is more complex than other technical control devices [7-10]

Consider the most characteristic features of the operator's activity, distinguishing it from the operation of the automatic control system (autopilot):

1. Discreteness of information perception. In the process of the control, the operator perceives information about the mismatch between the actual and required values of the vehicle motion parameters discretely and does not have the ability to continuously monitor it in the process of generating and implementing the control action.

2. The delay of response actions. The delay of the operator's reaction is much greater than the delay of the automatic control system devices.

3. Change in speed. Depending on the control task, the specific traffic conditions and the mismatch of the controlled parameters, the operator forms the control action with different speed and amplitude of the control deviation.

4. Different degree of adequacy of the size and form of the control action to this mismatch of the vehicle motion parameters is determined mainly by the acquired traffic driving skills, but also depends on other factors: the emotional state of the operator; traffic conditions; weather conditions, vehicle performance, etc.

5. The presence of sensitivity thresholds in the receptors of the operator. They exceed the values of similar characteristics of sensors of automatic devices and largely depend on the state of the operator. In addition, the sensitivity threshold increases as the intensity of the stimulus rises in accordance with the basic psychophysical law of Weber - Fechner.

6. The ability of the operator to predict the change of parameters, i.e. to anticipate the control result before the control actions.

The creation of the mathematical model that reflects the participation of the operator's intelligence in driving, the mechanism of acquiring driving skills and other aspects of the central nervous system, is associated with great difficulties due to lack of knowledge in this area.

Let us consider some approaches to the mathematical description of the operator actions, each of which has its advantages and disadvantages, takes into account the peculiarities of the operator actions. The application of different approaches depends on the specific task of management, in which the properties of a person become decisive.

The mathematical model of formation of the operator control actions reflects such psychophysical properties as the presence of absolute and differential sensitivity thresholds, takes into account the reaction delay and discreteness of information perception. This model can be used to model all software control tasks $[6,8,9]$

To form the control action of the operator in this mathematical model, we take the following conditions:

1. Depending on the control task, the operator generates the required steering wheel deviation. The operator develops this law of the steering wheel deviation in the process of acquiring driving skills. The control cycles, aimed at changing one or another parameter of movement when it deviates from a given value, are superimposed on the basic, required law of the steering wheel deviation.

2. The operator perceives the information discretely and does not continuously monitor it during the control cycle. If it is necessary to compensate for the deviation of some traffic 
from the predetermined one, then the feedback can be broken for the entire period of the control cycle.

3. The control action of the operator with respect to one direction of movement does not depend on the manoeuvres of the vehicle with respect to the other direction.

4. It is assumed that during the control cycle acceleration and speed change insignificantly.

5. The operator reacts to the mismatch of the actual parameter of the vehicle movement with the given one only if it is equal to or more than the threshold, i.e. perceived by his senses. time.

6. The operator performs the deviation of the steering wheel with a delay of his reaction

7. The operator forms the control action in such a way that the given parameter of the vehicle movement changes by a predetermined value monotonously, without casts. The time derivative of this parameter is zero at the beginning and end of the cycle, and in the middle part it reaches a maximum in absolute value.

\section{Research result}

Based on the above conditions, it is possible to calculate the necessary, when driving, deviation of the wheels and, accordingly, the steering wheel, knowing the required nature of the change in the vehicle motion parameters $[2,6,8,9,10]$

Let the operator needs to change some parameter of motion $t$ by the value of $\Delta$, in accordance with the accepted condition, the operator tends to the nature of the change $X(t)$, which is shown in Figure 1.

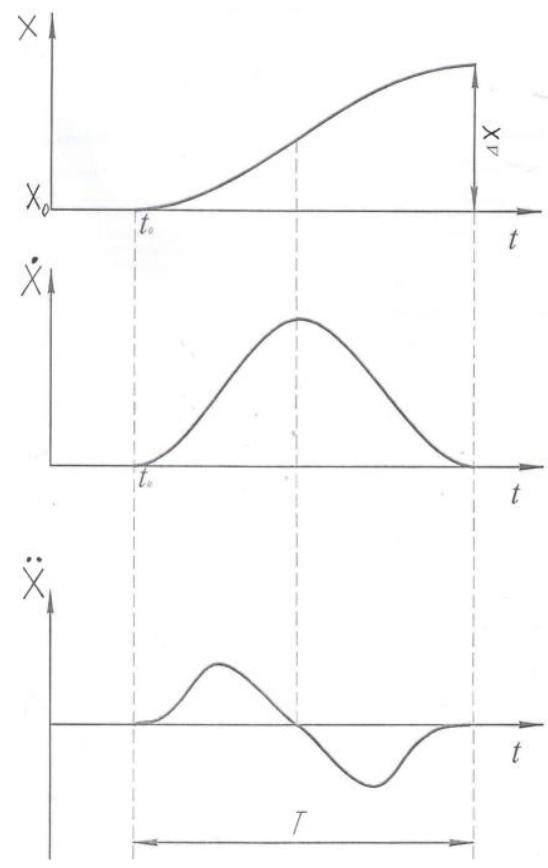

Fig.1. Diagram of change in the parameters of the vehicle movement

This change in the parameter $X(t)$ will correspond to the form of the first and second derivatives. If the parameter $\mathrm{X}$ is the position of the vehicle, then $\dot{X}$ and $\ddot{X}$ - speed and acceleration, respectively. 
As can be seen in Figure 1, it is convenient to approximate the change in derivative $\mathrm{X}$ by a sinusoidal function of time.

$$
\ddot{X}(\tau)=A \sin \omega t
$$

where $\mathrm{A}$ is the amplitude;

$\omega=2 \pi / T$ - frequency;

$\mathrm{T}$ is the control cycle period;

$\tau=t-t_{0}$; here $\mathrm{t}$ is the current time; $\mathrm{t}_{0}$ - the initial time of the control cycle, the current time.

Integrating equality (1), we obtain the functions describing $\dot{X}(\tau)$ and $X(\tau)$ :

$$
\begin{gathered}
\dot{X}(\tau)=\frac{1}{\omega}(1-\cos \omega \tau) \\
X(\tau)=\frac{A}{\omega}\left(\omega-\frac{1}{\omega} \sin \omega \tau\right)
\end{gathered}
$$

Further, using the motion equations and expressing the given parameter of motion $\mathrm{X}$ ( $\dot{X} \ddot{X}$ ) in them in accordance with formulas [1-3], we determine the necessary law of deviation of the control body.

If the control action is aimed at changing the first $\dot{X}$ or second $\ddot{X}$ derivative, then the formula (3) must be described respectively $\dot{X}(\tau)$ or $\ddot{X}(\tau)$. In this case, the change in the parameter $\dot{X}$ and $\ddot{X}$ is found by integration. The law of the control deviation is also determined by the substitution of the corresponding expressions for $\mathrm{X}, \dot{X}$ and $\ddot{X}$ into the equations of the vehicle motion.

Defined in this way the law of deviation of the control is summed with the need (lowfrequency or balancing), which is determined by the mode of motion and the control task and is introduced into the complete system of equations of the vehicle motion as a control function.

If a specific task of performing movement is set before the operator, for example, keeping the turning speed, another driving mode, then the given values of the vehicle motion parameters are introduced into the program. When reaching between one of them and its actual value of mismatch, exceeding the threshold value, the corresponding control action is calculated, aimed at preventing this mismatch.

\section{Conclusion}

The authors claim that if the operator is given a specific task to perform a certain traffic, for example, a traffic with a constant speed, a traffic with a subcritical rollover speed or another mode of traffic, then the program proposed by the authors should enter the specified values of the vehicle traffic parameters. When a mismatch exceeds the threshold between one of the set values of the traffic parameters and its actual value, the corresponding control effect is calculated to prevent this mismatch. These actions will allow you avoid a traffic accident while driving.

\section{References}

1. Yu.V. Buralev, Life Safety in Transport (M., Akadema, 2004)

2. V.N. Lukanina, Traffic safety: Training manual (M., Mashinbuilding, 1999) 
3. P.P. Konyukhovsky, Mathematical Methods of Operation Researches (M., 2002)

4. V.E. Kraskevich, K.Kh. Zelensky, V.I. Grechko, Numerical Methods in Engineering Researches (Kiev, High School, 1986)

5. A.A. Melnikov, Theory of Automatic Control of Technical Facilities of Vehicles and Tractors. Training manual for students of Higher Educational Institutions (M.: Publishing center Academy, 2003)

6. N.E. Sakovich, Safety of Transport Operations in Agricultural Production due to the Reduction of Accidents in Agricultural Transportation Technology (Thesis Doctor of Tech. Sciences, M.: 2013)

7. I.T. Tarasevich, Mathematical and Computer Modeling. Introductory Course (M.: URSS, 2001)

8. Ye.N. Khristoforov, Prevention of Accidents and Injuries of Drivers of Agricultural Vehicles by engineering activities (Thesis Doctor of Tech. Sciences., S-Pb, 2009)

9. V.S. Shkrabak, Ye.N. Khristoforov, N.E. Sakovich, Theory and Practice of Ensuring Traffic Safety in Agriculture // the Monography. - Bryansk.: publishing house BSAA, (2008).

10. V.V. Ambartsumyan, V.S. Shkrabak, V.V. Shkrabak, N.V. Smirnov, System Analysis of Traffic Safety (Saint-Petersburg,: Publishing House SPSAU, 2008) 